\title{
Cell Population Balance, Ensemble and Continuum Modeling Frameworks: Conditional Equivalence and Hybrid Approaches
}

\author{
Michail Stamatakis* \\ Department of Chemical and Biomolecular Engineering, Rice University, Houston, TX \\ 77005, USA
}

\begin{abstract}
The cell population balance, ensemble and continuum modeling frameworks are widely used for the mathematical description of microbial populations. Each of these approaches focuses on different aspects of the processes of growth, division and intracellular reaction occurrence. Therefore, each framework can output different information at different computational expense. Continuum models assume lumped biomasses, capture the dynamics of bulk intracellular concentrations and are easy to simulate. Ensemble models account for heterogeneity due to different initial conditions or kinetic constants, and are more computationally expensive. Finally, cell population balances capture the partitioning of the intracellular contents in detail, but can quickly become intractable, as the number of biochemical species taken into consideration increases. It is thus natural to ask whether one can adequately simulate cell populations with a simpler approach.

In this paper, starting from an $(n+1)$ cell population balance, for $n$ biochemical species and the cell volume, we investigate biologically plausible conditions, under which two exact hybrid models are derived. Provided that the conditions hold true, both hybrid models are exact but much simpler alternatives of the cell population balance; no approximation is involved. In the first, the evolution of the species concentrations is captured by an ensemble model and in the second by a continuum model. Both hybrid models also contain a 1dimensional population balance for the cell volume. This work should provide a guideline for choosing the modeling approach which is the most appropriate for the particular application.
\end{abstract}

\section{KEYWORDS}

Biological and biomolecular engineering; Continuum model; Ensemble; Formulation; Hybrid modeling; Mathematical modeling; Population balance

\footnotetext{
* Corresponding Author. Present Address: University of Delaware, Chemical Engineering Department, 238 Colburn Lab, 150 Academy Street, Newark, DE 19716. Phone: +1-302-831-8705, Fax: +1-302-831-1048, email:mstam@alumni.rice.edu
} 


\section{Introduction}

The cell population balance (CPB), the ensemble and the structured continuum modeling frameworks are widely used in Chemical Engineering for the mathematical description of the underlying biological processes occurring in microbial populations. Each of these frameworks is based on different assumptions, and thus, can output different information at different computational expense.

The structured continuum models attributed to Fredrickson (1976), utilize the assumption that the volume the cells occupy is lumped into a continuous and expanding biotic phase. Thus, one can formulate mass balances for the species concentrations taking into account the dilution effect due to cell growth. The continuum models consist of ordinary differential equations (ODEs) and are the easiest to simulate. However, they do not provide any information about the distribution of phenotypic characteristics on the population; rather they implicitly assume that all the cells share the same concentrations (those of the lumped biotic phase).

A more detailed description of the cell population is achieved with the use of the ensemble models, introduced by Shuler and coworkers (Domach and Shuler 1984; Ataai and Shuler 1985; see also Henson 2003 for a review on CPBs and ensemble models). These models are based on randomly perturbing the intracellular parameters or the initial conditions of a single cell model and simulating each cell individually, thereby creating an ensemble. Thus, one can obtain distributions for any of the cell properties into consideration. Cell division is typically not accounted for in these models, since the single cell models are written with respect to concentrations, which are intensive quantities and remain the same after division (unless chromosomal DNA species or species with low copy numbers are modeled; this point will be discussed in more detail later). The ensemble models give a more detailed description than that obtained from the structured continuum model, and can be used to simulate heterogeneous cell populations. However, the prohibitively slow dynamic simulation for large ensembles is a major disadvantage compared to the structure continuum models, especially since the distribution's resolution depends on the ensemble size.

A conceptually different approach, the CPB, was introduced in the ' 60 s by Fredrickson and coworkers (Eakman et al. 1966; Tsuchiya et al. 1966; Fredrickson et al. 1967). CPB models require single cell information and predict the distribution of phenotypic characteristics in the cell population level. In particular, the single cell information is summarized in the so-called physiological functions, which give the growth rate, the division rate and the partition probability density with respect to the physiological state of the cell. By assumption, the physiological state fully describes the cell's status, and is generally a vector, whose components can include the intracellular contents of chemical species as well as the morphometric characteristics of the cell. Therefore, the CPB models, can account for the different growth and division rates of the cells, as well as for unequal partitioning effects.

The high level of detail that these models can offer has motivated the development of computational algorithms for their numerical solution. Thus, Subramanian and Ramkrishna (1971) have used the method of weighted residuals with a successive approximation scheme to obtain solutions to a CBP for the cell mass. Mantzaris et al. (1999; 2001a; 2001b; 2001c) have developed and investigated the performance of different solution algorithms based on finite difference discretization schemes, as well as finite element and spectral methods. Test 
problems for 1-D and 2-D CPBs are solved with all methods, and also a 3-D problem is solved with finite differences. Furthermore, an efficient hierarchical discretization approach has been developed by Immanuel and Doyle III (2003). The approach is based on introducing two number densities, one at a coarse level and the other at a finer level of discretization. Subsequently, aggregation and breakage events are accounted for at the coarse level and growth at the finer level. The resulting two-tier algorithm has been employed to simulate 3-D population balances (Pinto et al. 2008) and 6-D CPBs (Pinto et al. 2007).

However, multidimensional CPB models with more than 6 variables are extremely difficult to solve, even with the current computational power available. Therefore, Monte Carlo (MC) algorithms were developed to simulate realizations of the underlying processes, and thus, compute phenotypic distributions, numbers of cells or any other desirable characteristic of the cell population. Shah et al. (1976) developed such an algorithm to simulate mass distribution dynamics which was later extended by Hatzis et al. (1995) to simulate the multi-staged growth of phagotrophic protozoa. These algorithms are computationally intensive because the number of cells in the population increases exponentially in time; thus, constant-number Monte Carlo algorithms were developed to simulate a constant number of cells which is a representative sample of the overall population. As a matter of fact, these algorithms do not follow the dynamics of population growth. Mantzaris (2006) has developed a variable number Monte-Carlo algorithm in which the sample size can be increased up to a maximum. Hence, a single cell can be simulated up to the point that there are $\mathrm{N}_{\max }$ offspring, from that point on the algorithm behaves as a constant-number (Smith and Matsoukas 1998).

In the aforementioned solution techniques, one aims at solving, or simulating stochastic paths, of the original CPB equation. Any errors originate from the discretization scheme employed in the solution algorithms, or the finite sample size in the $\mathrm{MC}$ algorithms. On the other hand, coarser descriptions of cell population dynamics have also been obtained and analyzed.

Zhang et al. have used nonlinear model reduction with proper orthogonal decomposition (2003) to investigate the transient behavior and the bifurcation structure of a CPB for glucose and ethanol fermentation in yeast. This approach is based on simulating the full order model, thereby obtaining spatiotemporal datasets, projecting these datasets onto a subspace properly chosen so that one retains most of the information (empirical eigenfunction basis extraction), and finally constructing a reduced model on that subspace.

Moreover, Mantzaris (2005) suggests that under the assumption of homogeneous cell populations, a continuum model can be formulated that corresponds to the CPB equation. This continuum model provides a coarser description of the cell population dynamics but neglects the heterogeneous nature of the cell population. Subsequently, Mantzaris (2005) compares the predictions of the continuum model with those of the CPB and attributes any differences to the effect of heterogeneity on the behavior of the cell population.

In this work we take a different approach. Instead of introducing approximate coarser models of the $\mathrm{CPB}$, we examine biologically plausible conditions under which exact hybrid models can be formulated. The latter models are mathematically equivalent to the original CPB when those conditions hold true. To this end, we start our discussion from the general CPB equation, taking into account volume and incorporating single cell growth. Thus, the population balance is written for an $(\mathrm{n}+1)$-dimensional state vector: $\mathrm{n}$ elements for the molar contents of $\mathrm{n}$ biochemical species, plus 1 element for the cell volume, all of which are 
extensive quantities. We subsequently highlight that there is some redundancy in the information contained in those variables, since each molar content contains information about the volume. This observation motivates the introduction of $n$ intensive quantities for the species concentrations, on the basis of which we derive a population balance equation for the concentrations and the volume. Subsequently, it is shown that the latter equation can be transformed under some conditions to hybrid models that consist of a 1-dimensional population balance for the cell volume and either an ensemble model or a continuum model for the $\mathrm{n}$ species concentrations. We provide a biological interpretation of these conditions and finally discuss the potential impact of this work.

\section{Methods}

\subsection{Modeling Frameworks}

Throughout the derivations of this work we will assume that the extracellular environment is homogeneous and that it spans a volume much larger than the total cell volume. Therefore, the extracellular species concentrations remain approximately constant.

\subsubsection{Population Balance}

The general CPB equation is a balance of expected cell numbers in the state space. It equates the rate of cell number change in a differential volume $\mathrm{d} \mathbf{z}$ of the state space with the inflows and outflows of cell numbers due to cell growth, reaction and division with stochastic partitioning. The state $\mathbf{z} \in \mathrm{G}$ is generally a vector that consists of the intracellular contents and morphometric characteristics of the cell (such as volume, membrane area, length etc.). The number density function $\mathrm{N}(\mathbf{z}, \mathrm{t})$ shows on average how many cells with state $\mathbf{z}$ exist in the population at time t. More precisely, $\mathrm{N}(\mathbf{z}, \mathrm{t}) \prod_{\mathrm{i}} \mathrm{d} \mathrm{z}_{\mathrm{i}}$ is the (expected) number of cells in the

differential volume $\mathrm{dz}$ of the state space at time t. The flow of $\mathrm{N}$ in the state space can be formulated in terms of the rates with which the cells undergo the processes of intracellular reaction/growth and division, $\mathbf{r}(\mathbf{z}, \mathbf{S})$ and $\Gamma(\mathbf{z}, \mathbf{S})$ respectively; the partitioning probability density function $\mathrm{p}(\mathbf{z} \mid \mathbf{y}, \mathbf{S})$; and the dilution rate $\mathrm{D}$, for continuous stirred tank bioreactors. In these expressions, $\mathbf{S}$ denotes the extracellular substrate concentrations. Further, the partitioning probability density specifies how the contents of the mother cell are partitioned into the two daughters upon division. Specifically, $\mathrm{p}(\mathbf{z} \mid \mathbf{y}, \mathbf{S})$ is the probability that a dividing cell at state $\mathbf{y}$ will give birth to two daughters having states $\mathbf{z}$ and $\mathbf{y}-\mathbf{z}$. Thus the CPB equation is (Fredrickson et al. 1967; Ramkrishna 2000):

$$
\begin{aligned}
\frac{\partial \mathrm{N}(\mathbf{z}, \mathrm{t})}{\partial \mathrm{t}}+\nabla_{\mathbf{z}} \cdot & {[\mathbf{r}(\mathbf{z}, \mathbf{S}) \mathrm{N}(\mathbf{z}, \mathrm{t})]+\Gamma(\mathbf{z}, \mathbf{S}) \mathrm{N}(\mathbf{z}, \mathrm{t})=} \\
& 2 \int_{\mathrm{G}} \Gamma(\mathbf{y}, \mathbf{S}) \mathrm{p}(\mathbf{z} \mid \mathbf{y}, \mathbf{S}) \mathrm{N}(\mathbf{y}, \mathrm{t}) \mathrm{d} \mathbf{y}-\mathrm{DN}(\mathbf{z}, \mathrm{t})
\end{aligned}
$$

subject to the regularity condition:

$$
\mathbf{r}(\mathbf{z}, \mathbf{S}) \mathrm{N}(\mathbf{z}, \mathrm{t})=0 \quad \mathbf{z} \in \partial \mathrm{G}
$$




\subsubsection{Ensemble model}

An ensemble model essentially tracks the timecourses of several single cell models which are simulated with different initial conditions or different parameters. Thus, let us assume that the single cell model for the concentrations $\mathbf{C}$ is written as:

$$
\frac{\mathrm{d} \mathbf{C}}{\mathrm{dt}}=\mathbf{f}(\mathbf{C}, \mathbf{S}, \boldsymbol{\kappa}, \mathrm{t})
$$

where $\kappa \in M$ is a random parameter set, determined once upon initiation of the simulation and $\mathbf{f}$ is a vector giving the rate of change of the concentrations. Let $\mathrm{p}_{0}(\boldsymbol{\kappa})$ be the probability density for $\kappa$, which accounts for perturbations in the parameters of the intracellular models. If we now define $\rho(\mathbf{C}, t)$ to be the normalized density of cells in the ensemble whose concentrations are within the differential volume $\mathrm{d} \mathbf{C}$ at time $\mathrm{t}$, then the ensemble model can be formulated as:

$\frac{\partial \rho(\mathbf{C}, \mathrm{t})}{\partial \mathrm{t}}+\int_{\mathrm{M}} \nabla \cdot[\mathbf{f}(\mathbf{C}, \mathbf{S}, \boldsymbol{\kappa}, \mathrm{t}) \rho(\mathbf{C}, \mathrm{t})] \mathbf{p}_{0}(\boldsymbol{\kappa}) \mathrm{d} \boldsymbol{\kappa}=0$

If we are interested in perturbing only the initial conditions having the parameter set fixed to $\boldsymbol{\kappa}_{\mathbf{0}}$ then the above equation simplifies to the advection equation:

$\frac{\partial \rho(\mathbf{C}, \mathrm{t})}{\partial \mathrm{t}}+\nabla \cdot\left[\mathbf{f}\left(\mathbf{C}, \mathbf{S}, \boldsymbol{\kappa}_{\mathbf{0}}, \mathrm{t}\right) \rho(\mathbf{C}, \mathrm{t})\right]=0$

\subsubsection{Continuum model}

The continuum model assumes that cells exist as a lumped biotic phase, and gives the temporal evolution of the intracellular concentrations, taking into account dilution due to cell growth. Thus, the concentrations of the intracellular species are changing according to:

$$
\frac{\mathrm{d} \mathbf{C}}{\mathrm{dt}}=\mathbf{q}(\mathbf{C}, \mathbf{S}, \boldsymbol{\kappa}, \mathrm{t})-\mu \mathbf{C}
$$

where $\mu$ is the average specific growth rate defined as:

$$
\mu=\overline{\left(\frac{1}{\mathrm{~V}} \frac{\mathrm{dV}}{\mathrm{dt}}\right)}
$$

and is usually assumed to be constant for these models.

\subsection{Transformation of the Population Balance Equation}

In order to transform the $\mathrm{CPB}$ to simpler hybrid models, we will start from equation (1) written for the molar contents of $\mathrm{n}$ species and the cell volume (all extensive variables). Subsequently, a coordinate transformation will be made, thereby allowing for concentrations (intensive variables) to appear in the equation along with the volume. We will further impose conditions on the physiological functions, which define the rates of cell growth, reaction, 
division and the partitioning mechanism. On the basis of these conditions, an Ansatz for the number density will be introduced, which will make possible the decoupling of the dynamics of the intensive variables (concentrations) versus the extensive one (volume). This decoupling will culminate in the formulation of hybrid models in which the dynamics of the extensive variable are captured with a single-variable population balance, and those of the intensive variables with either an ensemble or a continuum model.

The population balance equation in which the state of the cell consists of the species molar contents $\mathbf{x}$ and the volume $\mathrm{V}$ is written as:

$$
\begin{aligned}
& \frac{\partial \mathrm{N}(\mathbf{x}, \mathrm{V}, \mathrm{t})}{\partial \mathrm{t}}+\nabla_{\mathbf{x}} \cdot[\mathbf{r}(\mathbf{x}, \mathrm{V}, \mathbf{S}) \mathrm{N}(\mathbf{x}, \mathrm{V}, \mathrm{t})]+\frac{\partial}{\partial \mathrm{V}}[\mathrm{g}(\mathbf{x}, \mathrm{V}, \mathbf{S}) \mathrm{N}(\mathbf{x}, \mathrm{V}, \mathrm{t})]+ \\
& \quad+\Gamma(\mathbf{x}, \mathrm{V}, \mathbf{S}) \mathrm{N}(\mathbf{x}, \mathrm{V}, \mathrm{t}) \\
& \quad=2 \int_{\mathbb{R}_{0}^{+} \mathrm{G}} \Gamma(\mathbf{y}, \mathrm{W}, \mathbf{S}) \mathrm{p}(\mathbf{x}, \mathrm{V} \mid \mathbf{y}, \mathrm{W}, \mathbf{S}) \mathrm{N}(\mathbf{y}, \mathrm{W}, \mathrm{t}) \mathrm{d} \mathbf{y d} \mathrm{W}-\mathrm{D} \mathrm{N}(\mathbf{x}, \mathrm{V}, \mathrm{t})
\end{aligned}
$$

where $\mathbf{z}$ of equation (1) is now $(\mathbf{x}, \mathrm{V})$ and $\mathrm{g}(\mathbf{x}, \mathrm{V}, \mathbf{S})$ is the single cell growth rate. Vector $\mathbf{x}$ is an n-dimensional vector of non-negative real numbers $\mathbf{x} \in \mathrm{G}=\left(\mathbb{R}_{0}^{+}\right)^{\mathrm{n}}$. Thus, $\mathbf{z}$ is an $(\mathrm{n}+1)$ dimensional vector belonging to the non-negative closed orthant of $\mathbb{R}^{\mathrm{n}+1}$. Equation (8) is subject to the regularity conditions, which ensure that the number density does not leave the orthant just mentioned:

$$
\begin{aligned}
& \mathbf{r}(\mathbf{x}, V, S) N(\mathbf{x}, V, t)=\mathbf{0} \\
& g(\mathbf{x}, V, S) N(\mathbf{x}, V, t)=0 \\
& \text { when } x_{i}=0 \text { for any } i=1, \ldots, n, \text { or } V=0
\end{aligned}
$$

We observe that the molar contents $\mathbf{x}$ are extensive quantities, scaling linearly with the size of the cell as quantified by the volume $\mathrm{V}$. Thus, there is some redundancy of information in the way the state vector $\mathbf{z}$ has been set up. In order to eliminate this redundancy, it makes sense to define the intensive variables for the concentrations of the $\mathrm{n}$ species as follows:

$$
\mathbf{C}=\frac{\mathbf{x}}{\mathrm{V}}
$$

Note that for the transformation to be well defined, the volume must be strictly positive, $\mathrm{V} \in$ $(0, \infty)=\mathbb{R}^{+}$. Subsequently, we can make a coordinate transformation from the $(\mathbf{x}, \mathrm{V})$ space to the $(\mathbf{C}, \mathrm{V})$ space. The total differentials of $\mathbf{x}$ and $\mathbf{C}$ will be related as:

$$
(\mathrm{d} \mathbf{C}, \mathrm{dV})^{\mathrm{T}}=\mathbf{J} \cdot(\mathrm{d} \mathbf{x}, \mathrm{dV})^{\mathrm{T}}
$$

and the gradients with respect to $(\mathbf{x}, \mathrm{V})$ and $(\mathbf{C}, \mathrm{V})$ as:

$$
\nabla_{(\mathbf{C}, \mathrm{V})}=\left(\mathbf{J}^{\mathrm{T}}\right)^{-1} \nabla_{(\mathbf{x}, \mathrm{V})}
$$


where:

$$
\left(\mathbf{J}^{\mathrm{T}}\right)^{-1}=\left(\left[\begin{array}{ccccc}
\mathrm{V}^{-1} & 0 & \cdots & 0 & -\mathrm{x}_{1} \cdot \mathrm{V}^{-2} \\
0 & \mathrm{~V}^{-1} & \cdots & 0 & -\mathrm{x}_{2} \cdot \mathrm{V}^{-2} \\
\vdots & \vdots & \ddots & \vdots & \vdots \\
0 & 0 & \cdots & \mathrm{V}^{-1} & -\mathrm{x}_{\mathrm{n}} \cdot \mathrm{V}^{-2} \\
0 & 0 & \cdots & 0 & 1
\end{array}\right)^{\mathrm{T}}\right)^{-1}=\left[\begin{array}{ccccc}
\mathrm{V} & 0 & \cdots & 0 & 0 \\
0 & \mathrm{~V} & \cdots & 0 & 0 \\
\vdots & \vdots & \ddots & \vdots & \vdots \\
0 & 0 & \cdots & \mathrm{V} & 0 \\
\mathrm{C}_{1} & \mathrm{C}_{2} & \cdots & \mathrm{C}_{\mathrm{n}} & 1
\end{array}\right]
$$

Furthermore, the determinant of the Jacobian used in integration and probability/number density transformations is:

$|\mathbf{J}|=\mathrm{V}^{-\mathrm{n}}$

Therefore:

$$
\begin{aligned}
& \nabla_{\mathbf{x}}=\mathrm{V}^{-1} \nabla_{\mathbf{C}} \\
& \left.\frac{\partial}{\partial \mathrm{V}}\right|_{\mathbf{x}}=\left.\frac{\partial}{\partial \mathrm{V}}\right|_{\mathbf{C}}+\left.\left.\sum_{\mathrm{i}=1}^{\mathrm{n}} \frac{\partial \mathrm{C}_{\mathrm{i}}}{\partial \mathrm{V}}\right|_{\mathrm{C}_{j \neq i}} \frac{\partial}{\partial \mathrm{C}_{\mathrm{i}}}\right|_{\mathrm{C}_{j \neq i}, \mathrm{~V}}=\left.\frac{\partial}{\partial \mathrm{V}}\right|_{\mathrm{C}}-\left.\sum_{\mathrm{i}=1}^{\mathrm{n}} \frac{\mathrm{x}_{\mathrm{i}}}{\mathrm{V}^{2}} \frac{\partial}{\partial \mathrm{C}_{\mathrm{i}}}\right|_{\mathrm{C}_{\mathrm{j} \neq \mathrm{i}}, \mathrm{V}}=\left.\frac{\partial}{\partial \mathrm{V}}\right|_{\mathbf{C}}-\left.\sum_{\mathrm{i}=1}^{\mathrm{n}} \frac{\mathrm{C}_{\mathrm{i}}}{\mathrm{V}} \frac{\partial}{\partial \mathrm{C}_{\mathrm{i}}}\right|_{\mathrm{C}_{j \neq i}, \mathrm{~V}}
\end{aligned}
$$

and the number densities $\mathrm{N}$ and $\mathcal{N}$, pertaining to intracellular contents versus concentrations respectively, will be related as:

$$
\begin{aligned}
& \mathrm{N}(\mathbf{x}, \mathrm{V}, \mathrm{t})\left|\prod_{\mathrm{i}=1}^{\mathrm{n}} \mathrm{d} \mathrm{x}_{\mathrm{i}} \mathrm{dV}\right|=\mathcal{N}(\mathbf{C}, \mathrm{V}, \mathrm{t})\left|\prod_{\mathrm{i}=1}^{\mathrm{n}} \mathrm{dC}_{\mathrm{i}} \mathrm{dV}\right| \\
& \mathrm{N}(\mathbf{x}, \mathrm{V}, \mathrm{t})=|\mathbf{J}| \mathcal{N}(\mathbf{C}, \mathrm{V}, \mathrm{t})=\mathrm{V}^{-\mathrm{n}} \mathcal{N}(\mathbf{C}, \mathrm{V}, \mathrm{t})
\end{aligned}
$$

Similarly for the partition probability density:

$$
\mathrm{p}(\mathbf{x}, \mathrm{V} \mid \mathbf{y}, \mathrm{W}, \mathbf{S})=\mathrm{V}^{-\mathrm{n}} p(\mathbf{C}, \mathrm{V} \mid \mathbf{Y}, \mathrm{W}, \mathbf{S})
$$

Moreover, the relation between extensive (r) and intensive ( $r$ rate of reaction is:

$$
\mathbf{r}(\mathbf{x}, \mathrm{V}, \mathrm{S})=\mathrm{V} r(\mathbf{C}, \mathrm{V}, \mathrm{S})
$$

Finally, the new growth and division rate functions with respect to concentrations ( $g$ and $T$ respectively) are related to the previous ones ( $g$ and $\Gamma$ with respect to contents) as follows:

$$
\begin{aligned}
& \mathrm{g}(\mathbf{x}, \mathrm{V}, \mathbf{S})=g(\mathbf{C}, \mathrm{V}, \mathbf{S}) \\
& \Gamma(\mathbf{x}, \mathrm{V}, \mathbf{S})=T(\mathbf{C}, \mathrm{V}, \mathbf{S})
\end{aligned}
$$

Under the above transformations, the population balance is written: 


$$
\begin{aligned}
\mathrm{V}^{-\mathrm{n}} \frac{\partial \mathcal{N}(\mathbf{C}, \mathrm{V}, \mathrm{t})}{\partial \mathrm{t}}+\nabla_{\mathbf{C}} \cdot\left[\boldsymbol{r}(\mathbf{C}, \mathrm{V}, \mathbf{S}) \mathrm{V}^{-\mathrm{n}} \mathcal{N}(\mathbf{C}, \mathrm{V}, \mathrm{t})\right]+ \\
\quad-\sum_{\mathrm{i}=1}^{\mathrm{n}} \frac{\mathrm{C}_{\mathrm{i}}}{\mathrm{V}} \frac{\partial}{\partial \mathrm{C}_{\mathrm{i}}}\left[g(\mathbf{C}, \mathrm{V}, \mathbf{S}) \mathrm{V}^{-\mathrm{n}} \mathcal{N}(\mathbf{C}, \mathrm{V}, \mathrm{t})\right]+\frac{\partial}{\partial \mathrm{V}}\left[g(\mathbf{C}, \mathrm{V}, \mathbf{S}) \mathrm{V}^{-\mathrm{n}} \mathcal{N}(\mathbf{C}, \mathrm{V}, \mathrm{t})\right]+ \\
\quad+T(\mathbf{C}, \mathrm{V}, \mathbf{S}) \mathrm{V}^{-\mathrm{n}} \mathcal{N}(\mathbf{C}, \mathrm{V}, \mathrm{t}) \\
=2 \int_{\mathbb{R}^{+}} \int_{\Theta} T(\mathbf{Y}, \mathrm{W}, \mathbf{S}) \mathrm{V}^{-\mathrm{n}} p(\mathbf{C}, \mathrm{V} \mid \mathbf{Y}, \mathrm{W}, \mathbf{S}) \mathcal{N}(\mathbf{Y}, \mathrm{W}, \mathrm{t}) \mathrm{d} \mathbf{Y d W}-\mathrm{D} \mathrm{V}^{-\mathrm{n}} \mathcal{N}(\mathbf{C}, \mathrm{V}, \mathrm{t})
\end{aligned}
$$

and noting that $\mathrm{V} \in(0, \infty)$ the regularity conditions become:

$$
\begin{aligned}
& r(\mathbf{C}, \mathrm{V}, \mathbf{S}) \mathrm{V}^{-\mathrm{n}+1} \mathcal{N}(\mathbf{C}, \mathrm{V}, \mathrm{t})=\mathbf{0} \\
& g(\mathbf{C}, \mathrm{V}, \mathbf{S}) \mathrm{V}^{-\mathrm{n}} \mathcal{N}(\mathbf{C}, \mathrm{V}, \mathrm{t})=0
\end{aligned}
$$

when

$\mathrm{C}_{\mathrm{i}}=0$ for any $\mathrm{i}=1, \ldots, \mathrm{n}$, or $\mathrm{V} \rightarrow 0$

stating that $\mathcal{N}$ never leaves the non-negative orthant of $\mathbb{R}^{\mathrm{n}+1}$. On the basis of these conditions, in the following derivations we will neglect any terms expressing integration over boundaries.

We will now make an assumption that will allow us to treat the partitioning function.

Condition 1. Upon division, both daughters have the same species concentrations as their mother and random volumes possibly dependent on the state of the cell:

$$
p(\mathbf{C}, \mathrm{V} \mid \mathbf{Y}, \mathrm{W}, \mathbf{S})=\prod_{\mathrm{i}=1}^{\mathrm{n}} \delta\left(\mathrm{C}_{\mathrm{i}}-\mathrm{Y}_{\mathrm{i}}\right) \phi(\mathrm{V} \mid \mathbf{Y}, \mathrm{W}, \mathbf{S})
$$

where $\phi(\mathrm{V} \mid \mathbf{Y}, \mathrm{W}, \mathbf{S})$ pertains to volume partitioning. This assumption for equal concentrations is justified as follows: suppose that the mother cell of volume $\mathrm{V}_{\mathrm{m}}$ has $\xi_{\mathrm{m}}$ molecules of some species of interest. Upon division, the first daughter of volume $V_{d}$ will inherit $\xi_{d}$ molecules, and thus, the other daughter will have volume $\mathrm{V}_{\mathrm{m}}-\mathrm{V}_{\mathrm{d}}$ and content $\xi_{\mathrm{m}}-\xi_{\mathrm{d}}$. We assume that the partitioning mechanism is a sequence of $\xi_{\mathrm{m}}$ Bernoulli trials in which a molecule ends up in the first daughter with probability $\mathrm{V}_{\mathrm{d}} / \mathrm{V}_{\mathrm{m}}$. Hence, given the volume of the first daughter, the total amount of molecules it will inherit follows binomial distribution with number of trials equal to $\xi_{\mathrm{m}}$ and probability of success equal to $\mathrm{V}_{\mathrm{d}} / \mathrm{V}_{\mathrm{m}}$. From the behavior of the binomial distribution at the limit of infinite many trials (namely infinitely many molecules are partitioned) we deduce that:

$$
\frac{\xi_{d} \mid V_{d}}{\xi_{m}} \stackrel{\text { a.s. }}{\longrightarrow} \frac{V_{d}}{V_{m}}
$$


meaning that the random variable $\xi_{d}$ given $V_{d}$ converges almost surely to $V_{d} / V_{m}$. Therefore, the concentration of the species into consideration will be equal in mother and daughter cells. Note that the assumption fails if we are interested in the partitioning of a chromosomal DNA species, since these species are partitioned equally between the two daughters. This case is not of concern for this work. Thus:

$$
\begin{array}{r}
2 \int_{\mathbb{R}^{+}} \int_{\Theta} T(\mathbf{Y}, \mathrm{W}, \mathbf{S}) \mathrm{V}^{-\mathrm{n}} p(\mathbf{C}, \mathrm{V} \mid \mathbf{Y}, \mathrm{W}, \mathbf{S}) \mathcal{N}(\mathbf{Y}, \mathrm{W}, \mathrm{t}) \mathrm{d} \mathbf{Y d W}= \\
2 \mathrm{~V}^{-\mathrm{n}} \int_{\mathbb{R}^{+}} T(\mathbf{C}, \mathrm{W}, \mathbf{S}) \phi(\mathrm{V} \mid \mathbf{C}, \mathrm{W}, \mathbf{S}) \mathcal{N}(\mathbf{C}, \mathrm{W}, \mathrm{t}) \mathrm{dW}
\end{array}
$$

We further make some algebraic manipulations to simplify the divergence terms in equation (20):

$$
\begin{array}{r}
\sum_{\mathrm{i}=1}^{\mathrm{n}} \frac{\mathrm{C}_{\mathrm{i}}}{\mathrm{V}} \frac{\partial}{\partial \mathrm{C}_{\mathrm{i}}}\left[g(\mathbf{C}, \mathrm{V}, \mathbf{S}) \mathrm{V}^{-\mathrm{n}} \mathcal{N}(\mathbf{C}, \mathrm{V}, \mathrm{t})\right]=\mathrm{V}^{-\mathrm{n}-1} \mathbf{C} \cdot \nabla_{\mathbf{C}}[g(\mathbf{C}, \mathrm{V}, \mathbf{S}) \mathcal{N}(\mathbf{C}, \mathrm{V}, \mathrm{t})]= \\
\mathrm{V}^{-\mathrm{n}-1} \nabla_{\mathbf{C}} \cdot[g(\mathbf{C}, \mathrm{V}, \mathbf{S}) \mathbf{C} \mathcal{N}(\mathbf{C}, \mathrm{V}, \mathrm{t})]-\mathrm{V}^{-\mathrm{n}-1} g(\mathbf{C}, \mathrm{V}, \mathbf{S}) \mathcal{N}(\mathbf{C}, \mathrm{V}, \mathrm{t}) \nabla_{\mathbf{C}} \cdot \mathbf{C}
\end{array}
$$

and:

$$
\begin{aligned}
& \frac{\partial}{\partial \mathrm{V}}\left[g(\mathbf{C}, \mathrm{V}, \mathbf{S}) \mathrm{V}^{-\mathrm{n}} \mathcal{N}(\mathbf{C}, \mathrm{V}, \mathrm{t})\right]= \\
& \quad \mathrm{V}^{-\mathrm{n}} \frac{\partial}{\partial \mathrm{V}}[g(\mathbf{C}, \mathrm{V}, \mathbf{S}) \mathcal{N}(\mathbf{C}, \mathrm{V}, \mathrm{t})]-\mathrm{n} \mathrm{V}^{-\mathrm{n}-1} g(\mathbf{C}, \mathrm{V}, \mathbf{S}) \mathcal{N}(\mathbf{C}, \mathrm{V}, \mathrm{t})
\end{aligned}
$$

Noting that $\nabla_{\mathbf{C}} \cdot \mathbf{C}=\mathrm{n}$, using equations (25) and (26) with (20) and dividing by $\mathrm{V}^{-\mathrm{n}}$ gives:

$$
\begin{aligned}
\frac{\partial \mathcal{N}(\mathbf{C}, \mathrm{V}, \mathrm{t})}{\partial \mathrm{t}}+\nabla_{\mathbf{C}} \cdot\left\{\left[\boldsymbol{r}(\mathbf{C}, \mathrm{V}, \mathbf{S})-\frac{g(\mathbf{C}, \mathrm{V}, \mathbf{S})}{\mathrm{V}} \mathbf{C}\right] \mathcal{N}(\mathbf{C}, \mathrm{V}, \mathrm{t})\right\} \\
+\frac{\partial}{\partial \mathrm{V}}[g(\mathbf{C}, \mathrm{V}, \mathbf{S}) \mathcal{N}(\mathbf{C}, \mathrm{V}, \mathrm{t})]+T(\mathbf{C}, \mathrm{V}, \mathbf{S}) \mathcal{N}(\mathbf{C}, \mathrm{V}, \mathrm{t}) \\
=2 \int_{\mathbb{R}^{+}} T(\mathbf{C}, \mathrm{W}, \mathbf{S}) \phi(\mathrm{V} \mid \mathbf{C}, \mathrm{W}, \mathbf{S}) \mathcal{N}(\mathbf{C}, \mathrm{W}, \mathrm{t}) \mathrm{dW}-\mathrm{D} \mathcal{N}(\mathbf{C}, \mathrm{V}, \mathrm{t})
\end{aligned}
$$

Let us pause for a moment and interpret the above equation, which describes the change in the expected cell number density for state $(\mathbf{C}, \mathrm{V})$ at time $\mathrm{t}$. The equation expresses that this number density accepts a contribution from the cells that are growing and those whose concentrations change according to the intensive reaction rate reduced by the single cell dilution rate. Moreover, there is an outflow of cell number density corresponding to cells that are dividing from $\mathrm{V}$ to smaller volumes, and an inflow from bigger cells that divide giving cells in state $(\mathbf{C}, \mathrm{V})$. Equation (27) is also subject to the regularity condition (21).

Let us further make some assumptions that will allow us to decouple the dynamics of the number density over the intensive variables (concentrations, C) versus the extensive one (volume, V). 
Condition 2. The intensive reaction rate does not depend on the volume:

$r(\mathrm{C}, \mathrm{V}, \mathrm{S})=r(\mathrm{C}, \mathrm{S})$

This is a somewhat trivial assumption, since the intensive reaction rate is by definition volume-independent.

Condition 3. The growth rate depends only on the volume of the cell, and not on the substrate concentrations:

$g(\mathbf{C}, \mathrm{V}, \mathbf{S})=g(\mathrm{~V}, \mathbf{S})$

Imposing this condition is based on the premise that we can derive a phenomenological growth rate for the cell, using the cell size (volume) as the only piece of information. The biochemical network of reactions that we are studying does not influence growth. This decoupling of the reaction from the growth process is not an unreasonable practice. For example, artificial genetic networks are commonly modeled in isolation from the metabolic activities of the cell. However, if one's aim is to model for example the cell cycle, this decoupling will no longer be possible.

Condition 4. The division rate also depends only on the volume of the cell:

$T(\mathbf{C}, \mathrm{V}, \mathbf{S})=T(\mathrm{~V}, \mathbf{S})$

This assumption is justified similarly to the previous one. In particular, if one is studying a biochemical network of reactions the participating species of which are not involved in the division machinery of the cell, it makes sense to correlate the division rate with the size of the cell. Bigger cells have a higher propensity for division, in contrast to smaller ones.

The aforementioned assumptions allow us to introduce the following Ansatz that will allow us to eventually transform the CPB equation to simpler hybrids models.

Condition 5. Since reaction and growth are by assumption independent processes, we can decompose the number density function as a product of a volume-dependent part and a concentration-dependent part:

$\mathcal{N}(\mathbf{C}, \mathrm{V}, \mathrm{t})=n_{\mathrm{V}}(\mathrm{V}, \mathrm{t}) n_{\mathrm{C}}(\mathbf{C}, \mathrm{t}) \mathcal{N}_{0}(\mathrm{t})$

where $n_{\mathrm{V}}(\mathrm{V}, \mathrm{t})$ and $n_{\mathrm{C}}(\mathbf{C}, \mathrm{t})$ are normalized to unity and:

$\mathcal{N}_{0}(\mathrm{t})=\int_{\mathbb{R}^{+}} \int_{\Theta} \mathcal{N}(\mathbf{C}, \mathrm{V}, \mathrm{t}) \mathrm{d} \mathbf{C} \mathrm{dV}$

The variable $\mathcal{N}_{0}$ gives the expected number of cells in the population at time t. Its time derivative can be calculated by integrating equation (27) applying conditions 2-5: 
$\frac{\mathrm{d} \mathcal{N}_{0}(\mathrm{t})}{\mathrm{dt}}=\left(\int_{\mathbb{R}^{+}} T(\mathrm{~V}, \mathrm{~S}) n_{\mathrm{v}}(\mathrm{V}, \mathrm{t}) \mathrm{dV}-\mathrm{D}\right) \mathcal{N}_{0}(\mathrm{t})$

where we have used the normalization condition for $\phi$, written as:

$$
\int_{\mathbb{R}^{+}} \phi(\mathrm{V} \mid \mathbf{C}, \mathrm{W}, \mathbf{S}) \mathrm{dV}=1
$$

and have eliminated the boundary terms coming from the integration on the basis of the regularity conditions (21). Substitution of (33) back to (27) and using conditions 2-5 yields:

$$
\begin{aligned}
& \frac{\partial n_{\mathrm{V}}(\mathrm{V}, \mathrm{t}) n_{\mathrm{C}}(\mathbf{C}, \mathrm{t})}{\partial \mathrm{t}}+n_{\mathrm{V}}(\mathrm{V}, \mathrm{t}) n_{\mathrm{C}}(\mathbf{C}, \mathrm{t}) \int_{\mathbb{R}^{+}} T(\mathrm{~V}, \mathbf{S}) n_{\mathrm{V}}(\mathrm{V}, \mathrm{t}) \mathrm{dV} \\
& +\nabla_{\mathrm{C}} \cdot\left\{\left[\boldsymbol{r}(\mathbf{C}, \mathbf{S})-\frac{g(\mathrm{~V}, \mathbf{S})}{\mathrm{V}} \mathbf{C}\right] n_{\mathrm{V}}(\mathrm{V}, \mathrm{t}) n_{\mathrm{C}}(\mathbf{C}, \mathrm{t})\right\} \\
& +\frac{\partial}{\partial \mathrm{V}}\left[g(\mathrm{~V}, \mathbf{S}) n_{\mathrm{V}}(\mathrm{V}, \mathrm{t}) n_{\mathrm{C}}(\mathbf{C}, \mathrm{t})\right]+T(\mathrm{~V}, \mathbf{S}) n_{\mathrm{V}}(\mathrm{V}, \mathrm{t}) n_{\mathrm{C}}(\mathbf{C}, \mathrm{t}) \\
& =2 \int_{\mathbb{R}^{+}} T(\mathrm{~W}, \mathbf{S}) \phi(\mathrm{V} \mid \mathbf{C}, \mathrm{W}, \mathbf{S}) n_{\mathrm{V}}(\mathrm{W}, \mathrm{t}) n_{\mathrm{C}}(\mathbf{C}, \mathrm{t}) \mathrm{dW}
\end{aligned}
$$

We can now integrate equation (27) over all possible values of $\mathrm{V}$ in an attempt to eliminate the volume. After some algebraic manipulations we obtain:

$$
\frac{\partial n_{\mathrm{C}}(\mathbf{C}, \mathrm{t})}{\partial \mathrm{t}}+\nabla_{\mathbf{C}} \cdot\left\{\left[\boldsymbol{r}(\mathbf{C}, \mathbf{S})-\mathbf{C} \int_{\mathbb{R}^{+}} \frac{g(\mathrm{~V}, \mathbf{S})}{\mathrm{V}} n_{\mathrm{V}}(\mathrm{V}, \mathrm{t}) \mathrm{dV}\right] n_{\mathrm{C}}(\mathbf{C}, \mathrm{t})\right\}=0
$$

where we have used the normalization condition for $\phi$ (equation 34) and eliminated the boundary terms on the basis of the regularity conditions.

A similar integration procedure for equation (35), but this time over the possible values of $\mathbf{C}$, yields:

$$
\begin{gathered}
\frac{\partial n_{\mathrm{V}}(\mathrm{V}, \mathrm{t})}{\partial \mathrm{t}}+\frac{\partial}{\partial \mathrm{V}}\left[g(\mathrm{~V}, \mathrm{~S}) n_{\mathrm{V}}(\mathrm{V}, \mathrm{t})\right]+T(\mathrm{~V}, \mathrm{~S}) n_{\mathrm{V}}(\mathrm{V}, \mathrm{t}) \\
=2 \int_{\mathbb{R}^{+}} T(\mathrm{~W}, \mathrm{~S}) \phi(\mathrm{V} \mid \mathrm{W}, \mathrm{S}) n_{\mathrm{V}}(\mathrm{W}, \mathrm{t}) \mathrm{dW}+ \\
-n_{\mathrm{V}}(\mathrm{V}, \mathrm{t}) \int_{\mathbb{R}^{+}} T(\mathrm{~V}, \mathrm{~S}) n_{\mathrm{V}}(\mathrm{V}, \mathrm{t}) \mathrm{dV}
\end{gathered}
$$

where we have used the following condition:

Condition 6. The partitioning probability density $\phi(\mathrm{V} \mid \mathbf{C}, \mathrm{W}, \mathbf{S})$ has no concentration dependence: 
$\phi(\mathrm{V} \mid \mathbf{C}, \mathrm{W}, \mathbf{S})=\phi(\mathrm{V} \mid \mathrm{W}, \mathbf{S})$

This condition can be justified similarly to conditions 3 and 4 : $\phi(\mathrm{V} \mid \mathbf{C}, \mathrm{W}, \mathbf{S})$ essentially expresses the probability that a dividing cell with volume $\mathrm{W}$ and concentrations $\mathbf{C}$ will give birth to two daughters having volumes $\mathrm{V}$ and $\mathrm{W}-\mathrm{V}$. Assuming that the biochemical network under consideration can be considered independently of the partitioning mechanism then the volumes of the two daughters will only be probabilistically dependent on the volume of the mother cell.

To summarize what we have done in this section, starting from the population balance equation, we have imposed the following conditions: the concentrations of mother and daughter cells are the same (condition 1), the intensive reaction rate is volume independent (condition 2), the single cell growth rate, the division rate and the partitioning probability are concentration independent (conditions 3,4,6). These conditions allowed us to introduce an Ansatz (condition 5, equation 31) in which the dynamics of the number density over the volume and over the concentrations are decoupled. The resulting set of equations $(33,36,37)$ is a simpler but exact alternative for the population balance (20), in the sense that under the conditions just noted the two models are equivalent.

\subsection{Hybrid Models}

An inspection of equation (36) reveals that it resembles the advection equation with a time dependent velocity field due to the term:

$\mu(\mathbf{S}, \mathrm{t})=\int_{\mathbb{R}_{0}^{+}}\left[\frac{1}{\mathrm{~V}} g(\mathrm{~V}, \mathbf{S}) n_{\mathrm{V}}(\mathrm{V}, \mathrm{t})\right] \mathrm{dV}$

which is termed the average specific growth rate. The advection equation is essentially the ensemble model (5), where the single cell model has to be chosen appropriately.

Thus, we have transformed an $(\mathrm{n}+1)$-dimensional population balance to a hybrid model that consists of an ensemble model (equation 36) for the concentrations, a 1-dimensinoal population balance for the volumes (37) and an ODE for the average number of cells (33). Note that the ensemble model pertains to the intensive variables, whereas the population balance to the extensive one.

Solution of this hybrid model would entail the solution of the 1-dimensional population balance (37) to find $n_{\mathrm{V}}$, calculation of $\mu(\mathbf{S}, \mathrm{t})$ and the subsequent solution of the ensemble model (36) to find $n_{\mathrm{C}}$. Furthermore, if one wishes to know the average number of cells in time, equation (33) can also be solved since $n_{\mathrm{V}}$ is known.

In the following we will impose a few more conditions to further simplify and decouple the equations of the hybrid model.

Condition 7. The average specific growth rate is constant.

$$
g(\mathrm{~V}, \mathbf{S})=\mathrm{g}_{\mathrm{c}} \cdot \mathrm{V} \quad \Rightarrow \quad \mu(\mathbf{S}, \mathrm{t})=\mathrm{g}_{\mathrm{c}}
$$


This is equivalent to exponential growth rate at the single cell level. This expression has been advocated to be a realistic model describing the bacterial growth law between subsequent divisions (Cooper 1988). Then, equation (36) becomes the advection equation with constant (time independent) velocity field, thereby being a proper (in terms of closure) ensemble model:

$$
\frac{\partial n_{\mathrm{C}}(\mathbf{C}, \mathrm{t})}{\partial \mathrm{t}}+\nabla_{\mathbf{C}} \cdot\left\{\left[\boldsymbol{r}(\mathbf{C}, \mathbf{S})-\mathrm{g}_{\mathrm{c}} \mathbf{C}\right] n_{\mathrm{C}}(\mathbf{C}, \mathrm{t})\right\}=0
$$

The above equation can be solved by the method of characteristics (Evans 1998). The characteristic equation of (41) is:

$$
\frac{\mathrm{d} \mathbf{C}}{\mathrm{dt}}=r(\mathbf{C}, \mathbf{S})-\mathrm{g}_{\mathrm{c}} \mathbf{C}
$$

which is a structured continuum model as represented by equation (6). Thus, if the initial condition for $n_{\mathrm{C}}$ is a delta peak at $\mathbf{C}=\mathbf{C}_{\mathbf{0}}$, then the solution $n_{\mathrm{C}}(\mathbf{C}, \mathrm{t})$ will always be a delta peak whose motion is prescribed by the vector field appearing in the right hand side of equation (42). Therefore, the last condition we examine can be stated as follows:

Condition 8. The initial cell population is homogeneous. Mathematically, this means that at $\mathrm{t}=0$ the number density is a product of Dirac deltas:

$n_{\mathrm{C}}(\mathbf{C}, 0)=\prod_{\mathrm{i}=1}^{\mathrm{n}} \delta\left(\mathrm{C}_{\mathrm{i}}-\mathrm{C}_{\mathrm{i}, 0}\right)$

Note that we do not have to impose that the number density be a product of Dirac deltas for every $t \geq 0$. It is guaranteed by the convective nature of equation (41) and the initial condition (43). In this case, instead of solving the advection equation, it suffices to solve the ODE 42) subject to the initial condition:

$$
\mathbf{C}(0)=\mathbf{C}_{\mathbf{0}}
$$

Thus, under the extra conditions of constant specific growth rate and homogenous initial cell population (conditions 7 and 8 respectively) the ensemble model (equation 41) transforms to a structured continuum model. In this case, the hybrid model consists of equations $(33,37)$ and (42) subject to (44), and one can independently solve the system of ODEs (42) and the population balance for the volume (37). Subsequently equation (33) can be solved, since $n_{\mathrm{V}}$ is known, and the density functions $n_{\mathrm{C}}$ and $\mathcal{N}$ can be obtained from equations (31) and (43).

\section{Results}

We have shown that an $(\mathrm{n}+1)$-dimensional CPB can transform to simpler models under certain conditions. The first set of conditions calls for equal species concentrations in mother and daughter cells, size-independent intensive reaction rates, size-dependent but concentration-independent growth and division rates and partitioning mechanism. Under these conditions one can derive a hybrid model that consists of a 1-dimensional population balance and an ensemble model. If it is additionally assumed that the average specific growth 
rate is constant and that the initial cell population is homogeneous, then the hybrid model consists of 1-dimensional population balance and a structured continuum model.

\section{Discussion}

\subsection{Biological Plausibility of the Conditions}

It is important to note that these conditions are not too restrictive and it is reasonable to expect that under vegetative growth of bacterial populations most of them hold true. Yet, there are some subtleties associated with the imposing of these conditions and the physiological realm of validity for the population balance model.

In particular, condition 1 (equal concentrations in mother and daughters) was justified on the basis of the species into consideration existing in large copy numbers and following binomial partitioning. From a mathematical point of view, this justification might even be considered redundant, since the $\mathrm{CPB}$ assumes that the species contents are continuous variables, thereby restricting the realm of its validity to the thermodynamic limit of big system sizes. Thus, if one needs to take into account partitioning effects stemming from low copy numbers, one would have to take into account the discrete numbers of molecules, and thus, reformulate the population balance such that reaction occurrence be treated as a discrete process. There are real life applications though, in which the species of interest exist in high copy number, for example, in biotechnological applications where proteins are over-expressed to achieve maximal production.

The applicability of this condition (equal concentrations in mother and daughters), in the case of chromosomal DNA species deserves special attention. Since these species have to be partitioned equally between the two daughters, any case in which the latter have unequal volumes results in violating the condition. However, if the two daughter cells divide into two approximately equally-sized daughters, then one expects only small deviations from the case where the condition is exactly satisfied. In any case, the CPB does not take into account chromosomal DNA species in detail as it neglects DNA duplication altogether. Thus, the problem just discussed should not be of concern when writing a population balance pertaining to species such as enzymes and substrates.

We will not discuss condition 2, stating that the intensive reaction rate is not dependent on the volume as it is fundamentally based on the theory of chemical kinetics.

Instead, let us turn our attention to conditions 3,4 and 6 which allowed us to decouple the processes of growth and division from the chemical reactions. In the original formulation of the population balance (equation 8), the growth and division rates depend on both the species contents and the cell volume. Let us now assume that the species into consideration do not participate in or have any connection with the cell cycle or the division machinery of the cell. Then, it is reasonable to state that any correlation between species contents and rate of growth or division will be dictated by the size of the cell: a small newborn cell is not expected to divide anytime soon and grows slowly; on the contrary a bigger cell is about to divide and grows faster than the newborn cell. Additionally, the sizes of the daughter cells will depend on the size of the mother cell. As a measure of the cell size one could use any species content (extensive quantity) or the cellular volume, and then correlate this size to the rates of growth and division. However, introducing the intensive species concentrations, which do not scale with the cell size, sets the basis of decoupling growth and division from the species 
dynamics. Then the volume is the only measure of cell size and we explicitly state that the rates of growth and division as well as the partitioning probability density function are only volume-dependent (conditions 3, 4 and 6). Under these assumptions, we can state that the number density function can be decomposed as a product of a volume-dependent and a concentration-dependent part (condition 5).

The next requirement for constant specific growth rate (condition 7) was mathematically justified as a means for decoupling the ensemble model for the concentrations from the 1dimensional population balance for the volumes. Since the volume dependent growth rate affects the concentration dynamics through dilution, one needs to know the average specific growth rate in order to predict these dynamics. By assuming exponential single cell growth we are essentially simplifying the calculation of the average specific growth rate, which consequently turns out to be a constant. The exponential single cell growth law is not at all unreasonable from a biological perspective; in fact it has been stated that experimental data are indistinguishable from this model of cell growth (Cooper 1988).

The final condition, calling for identical species concentrations in the cells of the initial population (condition 8), is needed in order to arrive to a structured continuum model formulation. This is an admittedly questionable assumption; however, in cases where the distributions of the intracellular concentrations are narrow, it is expected that deviations from the ideal case of condition 8 will be small. Note that the homogeneity in the initial concentrations does not mean that the hybrid models will be unable to capture the heterogeneous nature of the intracellular molar contents (i.e. the extensive quantities of the system). This point is discussed below in detail.

\subsection{Capturing Heterogeneity}

In the hybrid models developed here, the coupling of the ensemble or continuum models for the intensive variables with the 1-D CPB for the extensive variable renders possible the capturing of cell population heterogeneity. For instance, consider a population in which all the cells have the same initial concentrations and the same or different volumes. For this and the next example we will assume that the conditions for the equivalence of the hybrid models and the original CPB apply. Then, one can use the continuum model to predict the cell concentrations, which will be identical for all cells at all times. Heterogeneity in the molar contents of the cells will then be a consequence of different cell volumes, a feature that will be accounted for by 1-D CPB of the hybrid model.

As a second example, suppose that the single cell dynamics are bistable and that the initial cell population consists of two subpopulations. Within each subpopulation, the cells have the same initial concentrations which will eventually drive them to different steady states. To predict the cell population dynamics in this case, one can use the hybrid model that consists of the ensemble model along with the 1-D CPB. In the ensemble model, the same ODE will be simulated twice with the two different initial conditions, producing the concentration response of the two subpopulations. In addition, the 1-D CPB will predict the distribution of size, as quantified by the cell volume. Thus, for this example, heterogeneity in the molar contents of the cells has two components which are successfully captured by the hybrid model: (i) the bistability in the single cell dynamics for the concentrations and (ii) the distribution of cell volumes. 
It is interesting to note that overlooking the effect of volume change during cell growth can lead to the formulation of models. For instance, Mantzaris (2005) has presented a continuum model which is claimed to correspond to the CPB under the assumption of no heterogeneity (equation 6 in Mantzaris 2005). Yet in the original CPB model considered in that paper, volume was never taken into account, and thus, a transformation between molar contents and concentrations is impossible. Consequently, the variables of the CPB and the alleged corresponding continuum model are either all extensive or all intensive, and it actually turns out that Mantzaris (2005) has written the population balance for concentrations which are intensive quantities (refer to equation 4 and pertinent text in Mantzaris 2005). However, the population balance formulation admits molar contents which are extensive and conserved, so that upon division, a mother cell with contents $\mathbf{y}$ gives birth to two daughters with contents $\mathbf{x}$ and $\mathbf{y}-\mathbf{x}$ respectively. Consequently, the equations in Mantzaris (2005) violate mass conservation, since, for any given species, the sum of the concentrations of the daughter cells is not equal to the concentration of the mother cell.

Further, to derive the corresponding continuum model, Mantzaris (2005) assumes that there exists a case where the number density function can be a product of Dirac deltas at all times (no heterogeneity). However, Mantzaris (2005) does not examine whether the population balance admits such a solution. In fact, one expects that for the number density function to be a product of Dirac deltas at all times, the cells must start from identical initial conditions, divide equally and in a synchronized manner. But then, the average of the number density function will change discontinuously at every division, and thus, its $1^{\text {st }}$ derivative will be undefined. Hence, an ODE for the average (the alleged "corresponding continuum model") is valid only within the time intervals of subsequent divisions. In contrast to this situation, note that in the derivation of our hybrid model, we encountered advection equation which guarantees that all the cells will have the same concentrations at all times, provided of course that they have the same initial concentrations. This is not necessarily true for the extensive quantities though: since volume dynamics are still captured by a 1-D CPB, it turns out that the molar contents in general will not be the same for all cells.

Finally, it turns out that the characterization of the resulting equation as the "corresponding continuum model" is inappropriate. Actually, the term that Mantzaris (2005) perceives as dilution due to cell proliferation (the latter being the increase of cell numbers in the population), is in fact irrelevant to the dilution term found in (Fredrickson 1976). The latter expresses dilution of species concentrations due to cell growth (namely, cell volume expansion). The former (the negative term in the right hand side of equation 4 in Mantzaris 2005) expresses the decrease in the average population content due to binary fission, which generates cells with smaller molar contents than the mother cell. Since the equations in Mantzaris (2005) neglect volume altogether, dilution due to single cell growth cannot be accounted for.

In view of the above observations, the marked difference between single cell and cell population dynamics shown by Mantzaris (2005) may be artificial, stemming from the superficial treatment of the extensive variables of the CPB.

\subsection{Ensemble Size and Population Size}

Thus, we have rigorously shown that under certain conditions, a CPB can be transformed into sets of equations consisting of ensemble or continuum models for the intensive variables (concentrations) and a single variable population balance for the extensive variable (cell 
volume). Yet, one might identify the following seaming paradox. In an ensemble model the ensemble size remains the same and in the continuum model, one essentially tracks a single average cell. Then, how is it possible to predict the population dynamics with these models, if the population size increases as division incessantly generates new cells?

The answer to this question lies in conditions 3-5 which make substantiate the decoupling of growth and division from the intracellular reactions. Suppose that our cell population at $t=0$ consists of two cells with different intracellular concentrations. For simplicity, also assume that the average specific growth rate is constant. Then, the intracellular species concentrations for all the offspring of the first cell will follow the deterministic path dictated by the continuum model. Thus, all offspring of the first cell will have the same concentrations although generally very different contents because they will have different volumes. The same applies to all the offspring of the second cell. Further, note that the growth rates of all the cells are on average the same, and the number density function in the population balance expresses expected normalized number of cells at a given state. Therefore, the fraction of offspring of the first cell with respect to total cells in the population will be constant at all times. The same applies to the fraction of offspring from the second cell. It follows that the extra information obtained by keeping track of all the offspring in the population is actually redundant. The argument can be generalized for any number of initial cells in the population and non-constant average specific growth rate (however, the requirement for only volumedependent growth rate still applies). This argument explains why we can use the ensemble or continuum models to simulate the cell population dynamics for the intensive variables under conditions $1-5$.

\section{Conclusions}

In this paper, biologically plausible conditions were sought, under which the CPB transforms to simpler hybrid models. The latter involve ensemble or continuum models for the intensive variables (intracellular species concentrations), and a 1-D CPB for the extensive variable (cell volume), thereby being much easier to solve than the original CPB. Moreover, the hybrid models have the great advantage of being exact in the sense that, when the conditions hold true, they are mathematically equivalent to the original CPB.

As a final remark, let us discuss how this work can provide a guideline for the choice of the framework that strikes the best balance between computational expense and level of detail for a particular application. In cases where the system of interest can be treated in isolation from the cell cycle and does not greatly affect the metabolic activities of the cell, one can safely use the ensemble or the continuum model formulations to accurately simulate the dynamics of the intracellular species concentrations. Examples of such systems are plasmids encoding for biochemical species that are not toxic to the cell and do not impose a significant burden in the cell's metabolic machinery. However, in cases where these criteria are not met, one needs to apply the more detailed population balance model with appropriately formulated physiological functions. The latter will have to express the differential growth and division rates and the asymmetry of partition as functions of the concentration (or content) of the species of interest, for instance in the case of a protein inhibiting cell division, or being overproduced to the extent that it slows down cell growth. 


\section{Acknowledgements}

The author would like to thank Konstantinos Spetsieris for stimulating discussions on the subject of population balance modeling and both Prof. Andreas Boudouvis and Konstantinos Spetsieris for reviewing the manuscript and contributing to its improvement. The author is grateful to the anonymous reviewers for their valuable contribution in improving the quality of this work. Financial support from the Kobayashi Fellowship Award (Chemical \& Biomolecular Engineering Department, Rice University) is also greatly appreciated.

\section{References}

Ataai, M. M. and M. L. Shuler (1985). "Simulation of CFSTR through development of a mathematical model for anaerobic growth of Escherichia coli cell population." Biotechnology and Bioengineering 27(7): 1051-1055.

Cooper, S. (1988). "What is the bacterial growth law during the division cycle?" Journal of Bacteriology 170(11): 5001-5005.

Domach, M. M. and M. L. Shuler (1984). "A finite representation model for an asynchronous culture of E. coli." Biotechnology and Bioengineering 26(8): 877-884.

Eakman, J. M., A. G. Fredrickson and H. M. Tsuchiya (1966). "Statistics and dynamics of microbial cell populations." Chemical Engineering Progress Symposium Series 62: 37-49.

Evans, L. C. (1998). Partial Differential Equations. Graduate Studies in Mathematics (19), American Mathematical Society.

Fredrickson (1976). "Formulation of Structured Growth Models." Biotechnology and Bioengineering XVIII: 1481-1486.

Fredrickson, A. G., D. Ramkrishna and H. M. Tsuchyia (1967). "Statistics and dynamics of prokaryotic cell populations." Mathematical Biosciences 1(3): 327-374.

Hatzis, C., F. Srienc and A. G. Fredrickson (1995). "Multistaged corpuscular models of microbial growth: Monte Carlo simulations." Biosystems 36(1): 19-35.

Henson, M. A. (2003). "Dynamic modeling of microbial cell populations." Current Opinion in Biotechnology 14(5): 460-467.

Immanuel, C. D. and F. J. Doyle III (2003). "Computationally efficient solution of population balance models incorporating nucleation, growth and coagulation: application to emulsion polymerization." Chemical Engineering Science 58(16): 3681-3698.

Mantzaris, N. V. (2005). "Single-cell gene-switching networks and heterogeneous cell population phenotypes." Computers \& Chemical Engineering 29(3): 631-643.

Mantzaris, N. V. (2006). "Stochastic and deterministic simulations of heterogeneous cell population dynamics." Journal of Theoretical Biology 241(3): 690-706.

Mantzaris, N. V., P. Daoutidis and F. Srienc (2001a). "Numerical solution of multi-variable cell population balance models: I. Finite difference methods." Computers \& Chemical Engineering 25: 1411-1440. 
Mantzaris, N. V., P. Daoutidis and F. Srienc (2001b). "Numerical solution of multi-variable cell population balance models: II. Spectral methods." Computers \& Chemical Engineering 25: 1441-1462.

Mantzaris, N. V., P. Daoutidis and F. Srienc (2001c). "Numerical solution of multi-variable cell population balance models: III. Finite element methods." Computers \& Chemical Engineering 25: 1463-1481.

Mantzaris, N. V., J.-J. Liou, P. Daoutidis and F. Srienc (1999). "Numerical solution of a mass structured cell population balance model in an environment of changing substrate concentration." Journal of Biotechnology 71(1-3): 157-174.

Pinto, M. A., C. D. Immanuel and F. J. Doyle III (2007). "A feasible solution technique for higher-dimensional population balance models." Computers \& Chemical Engineering 31(10): $1242-1256$

Pinto, M. A., C. D. Immanuel and F. J. D. III (2008). "A two-level discretisation algorithm for the efficient solution of higher-dimensional population balance models." Chemical Engineering Science 63(5): 1304-1314.

Ramkrishna, D. (2000). Population Balances: Theory and Applications to Particulate Systems in Engineering. San Diego, CA, Academic Press.

Shah, B. H., J. D. Borwanker and D. Ramkrishna (1976). "Monte Carlo simulation of microbial population growth." Mathematical Bioscience 31(1-2): 1-23.

Smith, M. and T. Matsoukas (1998). "Constant-number Monte Carlo simulation of population balances." Chemical Engineering Science 53(9): 1777-1786.

Subramanian, G. and D. Ramkrishna (1971). "On the solution of statistical models of cell populations." Mathematical Biosciences 10(1-2): 1-23

Tsuchiya, H. M., A. G. Fredrickson and R. Aris (1966). "Dynamics of microbial cell populations." Advances in Chemical Engineering 6: 125-206.

Zhang, Y., M. A. Henson and Y. G. Kevrekidis (2003). "Nonlinear model reduction for dynamic analysis of cell population models." Chemical Engineering Science 58(2): 429-445. 